\title{
Drug resistance in HIV patients with virological failure or slow virological response to antiretroviral therapy in Ethiopia
}

\author{
Alemseged Abdissa ${ }^{1,2^{*}}$, Daniel Yilma ${ }^{3}$, Jannik Fonager ${ }^{4}$, Anne M Audelin ${ }^{4}$, Lone H Christensen ${ }^{4}$, Mette F Olsen $^{5}$, \\ Markos Tesfaye ${ }^{6}$, Pernille Kaestel ${ }^{5}$, Tsinuel Girma ${ }^{7}$, Abraham Aseffa ${ }^{8}$, Henrik Friis $^{5}$, Court Pedersen ${ }^{2}$ \\ and Aase B Andersen ${ }^{2}$
}

\begin{abstract}
Background: The ongoing scale-up of antiretroviral therapy (ART) in sub-Saharan Africa has prompted the interest in surveillance of transmitted and acquired HIV drug resistance. Resistance data on virological failure and mutations in HIV infected populations initiating treatment in sub-Saharan Africa is sparse.

Methods: HIV viral load (VL) and resistance mutations pre-ART and after 6 months were determined in a prospective cohort study of ART-naïve HIV patients initiating first-line therapy in Jimma, Ethiopia. VL measurements were done at baseline and after 3 and 6 months. Genotypic HIV drug resistance (HIVDR) was performed on patients exhibiting virological failure (>1000 copies $/ \mathrm{mL}$ at 6 months) or slow virological response ( $>5000$ copies $/ \mathrm{mL}$ at 3 months and $<1000$ copies/mL at 6 months).

Results: Two hundred sixty five patients had VL data available at baseline and at 6 months. Virological failure was observed among 14 (5.3\%) participants out of 265 patients. Twelve samples were genotyped and six had HIV drug resistance (HIVDR) mutations at baseline. Among virological failures, 9/11 (81.8\%) harbored one or more HIVDR mutations at 6 months. The most frequent mutations were K103N and M184VI.
\end{abstract}

Conclusions: Our data confirm that the currently recommended first-line ART regimen is efficient in the vast majority of individuals initiating therapy in Jimma, Ethiopia eight years after the introduction of ART. However, the documented occurrence of transmitted resistance and accumulation of acquired HIVDR mutations among failing patients justify increased vigilance by improving the availability and systematic use of VL testing to monitor ART response, and underlines the need for rapid, inexpensive tests to identify the most common drug resistance mutations.

\section{Background}

In Ethiopia approximately 1.3 million people live with HIV and the estimated adult HIV prevalence is $1.5 \%$ [1]. During the past eight years, there has been a rapid scale-up of antiretroviral therapy (ART) which reached 250,000 adults in 2012, representing $86 \%$ of eligible patients $[2,3]$.

As ART roll-out continues in resource-limited settings, the risk of potential emergence of HIV drug resistance (HIVDR) is growing. This could be due to the absence of virological monitoring in routine clinical care and the use

\footnotetext{
* Correspondence: alemseged.abdisssa@ju.edu.et

${ }^{1}$ Department of Medical Laboratory Sciences \& Pathology, Jimma University, Jimma, Ethiopia

${ }^{2}$ Department of Infectious Diseases, Odense University Hospital, Odense, Denmark Full list of author information is available at the end of the article
}

of drugs with low genetic barriers such as non-nucleoside reverse transcriptase inhibitors [NNRTI] [4]. The use of nevirapine as monotherapy for preventing mother-tochild transmission may have further contributed to the problem [5].

Viral replication under suboptimal antiretroviral pressure leads to accumulation of resistance mutations, which limit future therapeutic choices [6] as mutations conferring resistance to one drug frequently confer cross resistance to other antiretroviral drugs within the same class [7]. Thus, it is essential for patient management to define the pattern of both primary and secondary resistance mutations.

The World Health Organization (WHO) recommends surveillance for transmitted HIVDR among antiretroviral- 
naïve patients and drug resistance mutations emerging during treatment in all countries involved in the ARV access programs $[8,9]$. Although transmitted and acquired HIV-1 drug resistance mutations have been well-described and longitudinally surveyed in high-income countries such as France [10], United States [11] and Denmark [12] there are few data on the subject in resource-limited settings.

The aim of our study was to assess the prevalence of virological failure and resistance mutations in patients initiating treatment in Ethiopia.

\section{Methods}

\section{Study setting}

The present study was part of a randomized controlled trial evaluating two nutritional supplements in adult patients initiating ART in Ethiopia, which was registered at www.controlled-trials.com (ISRCTN32453477). There were no differences in virological suppression among intervention groups (Olsen MF et al., submitted). The Ethiopian ART program was initiated in 2005 free of charge in hospitals and followed by the expansion to health centers in the periphery in 2007. Patients initiating ART at Jimma University Hospital, Jimma Health Center and Agaro Health Center were enrolled between July 2010 and August 2012. The first two centers are located in Jimma (a city of 150,000 inhabitants), with an urban site managing a total of approximately 1,617 patients on ART in Jimma University Hospital and 317 in Jimma Health center. The third centre is located in Agaro (a small town of 28,000 inhabitants) with approximately 240 patients on treatment.

\section{Study participants}

HIV-positive patients attending the ART clinics, who were ART naïve and eligible for ART according to the Ethiopian national guideline [13], were invited to participate in the study and followed for 6 months. Inclusion criteria were being adult ( $\geq 18$ years), living within approximately $50 \mathrm{~km}$ of Jimma, and consenting to participate. Pregnant or lactating women and patients with known previous use of ART were excluded. Participants gave informed consent and protocols were approved by Jimma University Ethics Review Board and National Research Ethics Committee of Ethiopia.

Decision to initiate treatment was made according to WHO criteria: stage IV irrespective of CD4 count, stage III if CD $4<350$, or CD $4<200$ cells $/ \mu$ lat any stage. The choice of ART combination was guided by national treatment guideline [13] and generally consisted of one of three first line regimens [Tenofovir (TDF) + Lamivudine $(3 \mathrm{TC})+$ Nevirapine (NVP)/Efavirenz (EFV); or Zidovudine $(\mathrm{AZT})+3 \mathrm{TC}+\mathrm{NVP} / \mathrm{EFV}$; or Stavudine $(\mathrm{d} 4 \mathrm{~T})+3 \mathrm{TC}+$ $\mathrm{NVP} / \mathrm{EFV}$. As part of the ART program, patients collected drugs every month for free. CD4 counts were monitored at
ART initiation and every 6 months. Viral load monitoring was performed as part of this study.

\section{Specimen collection and processing}

Whole blood was obtained for CD4 count and plasma samples were separated and stored at $-80^{\circ} \mathrm{C}$ until analyzed for HIV viral load and genotype. CD4 count and viral loads were measured at baseline and after 3 and 6 months on therapy. Genotypic HIVDR was done at baseline and after 6 months among selected participants based on viral load levels.

\section{Viral load and CD4 count}

HIV-1 viral load (VL) was quantified using a commercial PCR assay (RealTime HIV-1, Abbott Laboratories, Illinois, USA) and automated extraction system (m2000 Real Time System, Abbott Laboratories, Illinois, USA) was used. Extracted samples were amplified and detected on the m2000rt platform (Abbott Laboratories, Illinois, USA). Virological failure was defined as a confirmed VL $>1000$ copies/mL at 6 months and viral suppression is the success of attaining VL $<1000$ copies/mL at 6 months. Virological slow responders were defined as VL $\geq 5000$ copies/mL at 3 months but with viral suppression at 6 months. CD4 cells were enumerated using the Facscount ${ }^{\oplus}$ automated cell counter (Becton-Dickinson, Franklin Lakes, New Jersey, USA). Immunological failure was defined as a decline in CD4 count from baseline after 6 months of ART [14].

\section{Adherence to the ART}

Self-reported adherence to ART was documented monthly. In addition, efavirenz or nevirapine plasma concentrations were measured at 1 and 2 months using liquid chromatography-tandem mass spectrometry at Division of Clinical Pharmacology, university of Cape Town, South Africa. Limit of quantification was $0.01 \mu \mathrm{g} / \mathrm{ml}$ for both drugs.

\section{Resistance genotyping}

Plasma samples were shipped to Copenhagen, Denmark on dry ice for drug resistance mutation analysis at Statens Serum Institut (SSI, Copenhagen DK). Amplification of the pol gene was performed in two steps using SuperScript $^{\text {Ts }}$ III One-Step RT-PCR System with Platinum ${ }^{\bullet}$ Taq High Fidelity (Invitrogen) in accordance with manufacturer's instructions. First cDNA synthesis and first PCR was performed using the primers JA203 [15] and IN3 [16] at the following conditions: $52^{\circ} \mathrm{C} 30 \mathrm{~min}, 94^{\circ} \mathrm{C}, 2 \mathrm{~min}$ followed by 40 cycles of: $94^{\circ} \mathrm{C}, 15 \mathrm{sec} ; 60^{\circ} \mathrm{C}, 45 \mathrm{sec} ; 66^{\circ} \mathrm{C}$, $3 \mathrm{~min}$ and a final extension at: $66^{\circ} \mathrm{C}$ for $10 \mathrm{~min}$. The second nested-PCR was performed using the Expand High Fidelity PCR System (Roche) in accordance with manufacturer's instructions and with the primers JA204 [15] and ABI1_R [17] at the following conditions: $94^{\circ} \mathrm{C}, 2$ 
min followed by 35 cycles of: $94^{\circ} \mathrm{C}, 30 \mathrm{sec} ; 60^{\circ} \mathrm{C}, 30 \mathrm{sec}$; $72^{\circ} \mathrm{C}, 60 \mathrm{sec}$, and a final extension at: $72^{\circ} \mathrm{C}$ for $7 \mathrm{~min}$, yielding a PCR fragment of $1449 \mathrm{bp}$. PCR products were prepared for sequencing using Illustra ${ }^{\mathrm{TM}}$ ExoProStar 1 step (GE Healthcare) and sequencing reactions were carried out using the BigDye Terminator version 1.1 (Applied Biosystems) and the sequencing primers:

INH-A:CTTCAGAGCAGACCAGAGCCAA, INH-B:GTTAAACAATGGCCATTGACAGA, INH-D:CAGRCYGGAGCCAACAGC,INH-C:GATG GAAAGGATCACCRGCAATA, INH-F:TTGGGCCAT CCATTCCTGG,

\section{INH-G:CCATCCCTGTGGAAGCACAT}

INH-H:TTCTGTATTTCTGCTAYTAAGTCTTTTG.

Sequencing was performed on an ABI 377 DNA Sequencer (Applied Biosystems) and sequence assembly and analysis was performed in BioNumerics v. 6.6 (Applied Maths, Sint-Martens-Latem, Belgium). For six of the 29 analyzed samples (Id \#_month; 25_6, 146_6, 301_6, 314_6, 360_6 and 25_0), sequences were obtained using the ViroSeq HIV-1 genotyping System v. 2 (Abbott Diagnostics, Foster City, CA) in accordance with manufacturer's instructions.

\section{Drug resistance mutation analysis and statistical methods}

For each sequence, the resistance profile was calculated using the current HIVdb algorithm [18] at Stanford's HIV genotypic resistance profile (http://sierra2.stanford.edu/ sierra/servlet/JSierra?action=sequenceInput) and as implemented in BioNumerics v. 6.6.

Statistical analysis was done using STATA/IC version 11.2 (StataCorp LP, College Station, USA). Differences in means, medians and proportions between men and women were tested using Pearson Chi-square test and the Fisher's exact test. P-values $<0.05$ were considered significant.

\section{Sequences submitted to GenBank}

Sequences obtained in this study were submitted to GenBank under the following accession numbers: KJ561 122-KJ561136 (baseline samples) and KJ561137-KJ5611 50 (6 months samples).

\section{Results}

\section{Participants and their baseline characteristics}

A total of 312 adult patients on first-line ART were enrolled into the study. Of these, 281 and 273 had reached 3 and 6 months of follow up, respectively, while the rest dropped out for various reasons including withdrawal of consent, lost to follow up or death. A total of 275 participants had VL results available at 3 months and 265 at 6 months. At ART initiation, the 265 participants retained for 6 months of follow-up did not differ significantly from the rest of participants $(n=47)$, in terms of baseline characteristics such as age, BMI, $\mathrm{VL}$ and WHO stage. The mean $( \pm \mathrm{SD})$ age was $33.0( \pm 8.8)$ years and $67 \%$ were women (Table 1). A combination of tenofovir (TDF), lamuvidine (3TC) and efavirenz (EFV) or nevirapine (NVP) was the most commonly (82.6\%) prescribed first-line regimen. The other first-line regimens contained zidovudine (AZT; 14.3\%) or stavudine (d4T; 3\%) instead of TDF.

\section{Virological outcomes and immunological criteria}

Virological failure was observed in 14 (5.3\%) of the participants. Three of the participants with good viral suppression at 6 months had a slow response. These participants (Id \# 146, 301 and 314) had 184878, 6849 and 125562 copies/mL at 3 months, respectively (Table 2). It was found that $158 / 275$ (57.5\%) and 233/265 (87.9\%) of the participants achieved $\mathrm{VL}<40$ copies $/ \mathrm{mL}$ at 3 and 6 months respectively. Patients experiencing virological failure had significantly lower CD4 count at 6 months mean $( \pm$ SD $)=169( \pm 85)$ compared to those with successful viral suppression $313( \pm 131) ; \mathrm{P}=0.002)$. However, only four of the 14 participants with virological failure (28.6\%) experienced immunological failure at 6 month (Table 2) and, 22 of 251 (8.7\%) participants with virological suppression experienced immunological failure.

\section{HIVDR mutations at baseline}

HIVDR genotyping was performed in pre-ART plasma specimens from 12 participants with virological failure. All patients were infected with HIV subtype C. Resistances to NNRTIs were identified in six of $12(50 \%)$ and none were resistant to NRTI. Mutations detected in three of the patients are associated with high level resistance. Five participants with HIVDR mutations at ART initiation received ART regimens that were only partially active (Additional file 1: Table S1). During the 6 months of follow-up, none of these patients were switched to a fully active first-line or second-line regimen.

A total of seven patients had HIVDR mutations detectable in the baseline samples; one mutation (V90IV) confers no resistance (Id \# 339). The K103N/X was the most common mutation observed. However, one participant (Id \# 25) had a mutation (A98G) that confers resistance to nevirapine. This patient was treated with a supposedly active efavirenz based regimen and the mutation had disappeared at 6 months (Additional file 1: Table S1).

\section{HIVDR mutations at 6 months}

HIVDR genotyping was performed in plasma from 11 participants with virological failure at 6 months. Nine of them (81.8\%) harboured mutations that cause high level resistance to NNRTIs. Six participants had the mutations at baseline, and three (Id \# 9, 339 and 346) acquired additional mutations during follow-up causing resistance to NRTI. Three of the participants with HIVDR had no resistance at baseline but acquired resistance mutations during the 
Table 1 Baseline characteristics of HIV-1 positive participants treated with first-line ART regiments in Jimma, Ethiopia ( $\mathbf{n}=312)$

\begin{tabular}{|c|c|c|c|c|}
\hline & Women $(n=179)$ & Men $(n=86)$ & Total $(n=265)$ & Excluded, did not complete 6 months $(n=47)$ \\
\hline Age, y & $31.0 \pm 8.0$ & $38.0 \pm 9.0$ & $33.0 \pm 8.8$ & 32.2 \\
\hline Body mass index (BMI), $\mathrm{kg} / \mathrm{m}^{2}$ & $19.6 \pm 2.5$ & $19.1 \pm 1.8$ & $19.4 \pm 2.3$ & $19.5 \pm 2.5$ \\
\hline CD4 count at enrollment, cells/ul & $189 \pm 113$ & $194 \pm 113$ & $190.7 \pm 113$ & $181 \pm 105$ \\
\hline Viral load, log(copies/mL ) & $4.7 \pm 1.0$ & $4.6 \pm 0.9$ & $4.7 \pm 1.0$ & $4.8 \pm 0.9$ \\
\hline \multicolumn{5}{|l|}{ WHO stage } \\
\hline Stage I & $63(35.2)$ & $23(26.7)$ & $86(32.5)$ & $11(23.4)$ \\
\hline Stage $\|$ & $47(26.3)$ & $32(37.2)$ & $79(29.8)$ & $12(25.5)$ \\
\hline Stage III & $53(29.6)$ & $26(30.2)$ & $79(29.8)$ & $19(40.4)$ \\
\hline Stage IV & $16(8.9)$ & $5(5.8)$ & $21(7.9)$ & $5(10.6)$ \\
\hline \multicolumn{5}{|l|}{ ART regimen } \\
\hline TDF/3TC/EFV & $113(63.1)$ & 77 (89.5) & $190(71.7)$ & $33(70.2)$ \\
\hline AZT/3TC/EFV & $2(1.1)$ & $1(1.2)$ & $3(1.1)$ & $1(2.1)$ \\
\hline TDF/3TC/NVP & $27(15.1)$ & $2(2.3)$ & $29(10.9)$ & $3(5.7)$ \\
\hline AZT/3TC/NVP & $31(17.3)$ & $4(4.7)$ & $35(13.2)$ & $10(18.9)$ \\
\hline Other* & $6(3.4)$ & $2(2.3)$ & $8(3.0)$ & $0(0.0)$ \\
\hline
\end{tabular}

*Other regimen includes mainly D4T and NVP based treatment. Data are mean \pm SD or $n(\%)$.

course of treatment and two of them became resistant to both NNRTIs and NRTIs. HIVDR genotyping could not be performed for 3 participants (Id \# 164, 354 and 373) due to failed sequencing attempt or sample inadequacy (Additional file 1: Table S1).

Of the three slow responders, one (Id \# 301) was resistant to all three drugs prescribed, while we found no evidence of HIVDR in the other two participants at 6 months (Id \# 146 and 314) (Additional file 1: Table S1). The former had pre-existing mutation and acquired additional mutations developing high level resistance to all the three antiretroviral drugs during the course of treatment (Additional file 1: Table S1).

All cases of HIVDR involved at least one NNRTI mutation, with the K103N (3/9) being the most frequently observed. Combined NNRTI and NRTI mutations were seen in four of nine (44.4\%) participants and involved M184V/I in all cases and K65R and D67G occurred in one participant in addition to the M184I mutation. A K65R mutation was also detected in a slow responding patient. While five of 11 participants with virological failure received ART regimens that were not or only partially active, the other four became resistant during the therapy in spite of a fully active ART regimens initiated at baseline (Additional file 1: Table S1).

A total of four participants, two from the virologicaly failing group and another two from the slow responders had no HIVDR mutations at 6 months. Self reported adherence data indicated that all participants with genotype data had good treatment adherence except two of the slow responders (Id \# 301 and 314). Measurements of
NNRTIs plasma concentrations indicated two out of nine participants with resistance mutations at 6 months (Id \# 213 and 269) and one participant with no resistance mutation (Id \# 357) had a drug concentration below the limit of quantification at 1 and 2 months (Table 2).

\section{Discussion}

This report describes virological failure or slow virological response and associated HIVDR mutations in a cohort of HIV patients initiating first-line regimen in the Ethiopian national ART roll out program. The study revealed a virological failure rate of $5.3 \%$ and transmitted resistance among 6/12 of the virological failures in this cohort. Resistance mutations were detected in 9/11 (81.8\%) of the patients failing treatment at 6 months. Although the virological failure rate was low, the transmitted resistance documented in this study, in a setting where ART has only been available for eight years is alarming.

A strength of this study is that patients were closely followed in a clinical trial unit for regular clinical and laboratory monitoring at 3 and 6 months, leading to reliable identification of virological failure and minimizing losses-to-follow-up. The potential limitation is that the patients participated in a nutritional intervention study in which they were motivated to come to the ART clinic and perhaps had better ART adherence, compared to patients accessing treatment in a regular setting. The drug resistance mutation analysis was done only on virological failures because of limited resource. Thus, the actual rate of transmitted drug resistance was not estimated. 
Table 2 Characteristics of participants with virological failure and slow virological responders

\begin{tabular}{|c|c|c|c|c|c|c|c|c|c|c|}
\hline \multirow[b]{2}{*}{ Patient Id } & \multirow[b]{2}{*}{ Age } & \multirow[b]{2}{*}{ Sex } & \multicolumn{2}{|c|}{ Viral load (copies/mL) } & \multicolumn{2}{|c|}{ 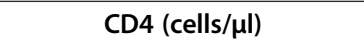 } & \multirow[b]{2}{*}{ Decline in CD4 count } & \multirow[b]{2}{*}{$\begin{array}{l}\text { NNRTI conc, } \mu \mathrm{g} / \mathrm{ml} \\
\text { at } 1 \text { month }\end{array}$} & \multirow[b]{2}{*}{$\begin{array}{l}\text { NNRTI conc, } \mu \mathrm{g} / \mathrm{ml} \\
\text { at } 2 \text { months }\end{array}$} & \multirow[b]{2}{*}{$\begin{array}{l}>95 \% \text { reported } \\
\text { adherence }\end{array}$} \\
\hline & & & At 3 months & At 6 months & At baseline & At 6 months & & & & \\
\hline \multicolumn{11}{|c|}{ Virological failures } \\
\hline 9 & 40 & M & 16816 & 7703 & 124 & 235 & No & 2.16 & 2.27 & Yes \\
\hline 25 & 39 & M & 54 & 116373 & 114 & 163 & No & 4.6 & 4.64 & Yes \\
\hline 157 & 47 & M & 91377 & 51498 & 41 & 153 & No & 2.9 & Missing & Yes \\
\hline 164 & 22 & $\mathrm{~F}$ & $<40$ & 11420 & 127 & 178 & No & 3.88 & 1.39 & Yes \\
\hline 213 & 35 & $\mathrm{~F}$ & 767340 & 29427 & 114 & 251 & No & $<0.01$ & $<0.01$ & Yes \\
\hline 243 & 26 & $\mathrm{~F}$ & $<40$ & 163259 & 207 & 116 & Yes & 8.7 & 8.04 & Yes \\
\hline 269 & 30 & $\mathrm{~F}$ & 86463 & 66498 & 207 & 331 & No & $<0.01$ & 0.01 & Yes \\
\hline 300 & 50 & $\mathrm{~F}$ & Undetectable & 28038 & 119 & 199 & No & Missing & 2.48 & Yes \\
\hline 339 & 30 & $\mathrm{~F}$ & 42735 & 381889 & 19 & 81 & No & 10.9 & 10.1 & Yes \\
\hline 346 & 25 & $\mathrm{~F}$ & Missing & 2002 & 225 & 357 & No & 5.34 & Missing & Yes \\
\hline 354 & 40 & M & 12237 & 30252 & 137 & 122 & Yes & 2.32 & 2.94 & Yes \\
\hline 357 & 30 & $\mathrm{~F}$ & 24931 & 143175 & 221 & 240 & No & $<0.01$ & $<0.01$ & Yes \\
\hline 360 & 32 & $\mathrm{~F}$ & 7289 & 111647 & 287 & 192 & Yes & 18.6 & Missing & Yes \\
\hline 373 & 34 & $\mathrm{~F}$ & 344294 & 78504 & 48 & 5 & Yes & $<0.01$ & $<0.01$ & Yes \\
\hline \multicolumn{11}{|c|}{ Slow virological responders } \\
\hline 146 & 20 & $\mathrm{~F}$ & 184878 & 141 & 131 & 360 & No & 4.42 & 6.54 & Yes \\
\hline 301 & 30 & $\mathrm{~F}$ & 6849 & 640 & 79 & 214 & No & 4.33 & Missing & No \\
\hline 314 & 50 & F & 125562 & 379 & 72 & 72 & No & Missing & $<0.01$ & No \\
\hline
\end{tabular}


Comparison of virological failure and HIVDR mutation rates obtained from different studies must be interpreted with caution, since threshold and duration of ART at time of failure varies. In a review from Sub-Saharan Africa countries (in which Ethiopia was not included), it was reported that virological success (defined by a VL $<400$ copies/mL) rates after 6 months of ART was 78\% [19]. In fact, $67 \%$ of the patients obtained viral suppression when applying lower cut-off values for success (VL less than 40 or 50 copies $/ \mathrm{mL}$ ). In this study, VL $<40$ copies $/ \mathrm{mL}$ at 6 months was achieved in $87.9 \%$ of participants. Thus, compared with data from other Sub-Saharan African countries, the success rates found in the present study was higher using the same threshold.

The patterns of HIVDR mutations among the patients failing treatment in the present study differed from those described in previous two studies conducted in Ethiopia. In a study conducted in Gondar, only two mutations (V75I and G190A) were detected among 92 ART-naïve patients in 2003, before ART roll out [20]. Another investigation in Addis Ababa did not find any mutations among 39 women attending antenatal care units in 2005 [21]. In our study however, several mutations, which may cause moderate to high level of resistance to the NNRTIs and NRTIs were detected, indicating a changing pattern and a rise in transmitted resistance to antiretroviral drugs in Ethiopia.

Although derived from a small study, it is noticeable that 9/11 (81.8\%) study participants genotyped at treatment failure had acquired or transmitted HIVDR, which is higher than the $63.7 \%$ reported from a study of 2000 HIV positive patients initiating first line ART using the WHO approach in other east African countries between 2006 and 2010 [22]. In three of nine participants with HIVDR mutations at baseline, exposure to a failing regimen during 6 months was associated with the emergence of additional mutations under drug pressure, including M184V/I. Among participants failing therapy and genotyped, two did not show any evidence of HIVDR mutations. Both participants reported very good adherence. However, efavirenz plasma concentrations in one of the participants were below limit of quantification at both 1 and 2 months visits. Although, reduced absorption could not be ruled out, the virological failure in this participant is likely due to undisclosed poor adherence [23].

Overall, the NRTI and NNRTI transmitted and acquired mutation patterns that were identified were consistent with previous reports in similar settings $[19,24,25]$. The most frequent NRTI mutation (M184V) and NNRTI mutations (K103N) described in this study are known to be common in cases of treatment failure [22]. The presence of transmitted resistance mutations has important implications for clinical management of HIV patients $[26,27]$. In addition, an immunological criterion alone has limitations to detect virological failures as shown in this study. These raise concerns about the routine use of the first-line regimens without access to viral load determination in Ethiopia.

Interestingly, one of the three patients exhibiting slow virological response had high level resistance to all the three drugs, although the VL was below 1000 copies/mL at 6 months. This may be due to the K65R mutation that can impair replicative fitness of the virus [28]. However, one of the virological failure also carried K65R; and the difference in virological outcome of these patients could be due to modulation of fitness cost by mutational interactions [29]. It is also interesting to note that many of the failing patients $(70 \%)$ had VL $>5000$ copies $/ \mathrm{mL}$ at 3 months in contrast to those with successful viral suppression, which highlights the potential of this criterion to identify virological failure and HIVDR earlier.

\section{Conclusions}

In summary, we report virological success and resistance rates and pattern of HIVDR mutations among Ethiopian patients initiating first-line ART. After eight years of largescale ART introduction, this survey demonstrated a low rate of virological failure. However, major mutations including K103N and M184V were identified, indicating that access to virological monitoring is of paramount importance to prevent inappropriate drug switches and preserve efficacy of ART in resource constrained countries. Moreover, the findings underscore the need for rapid and inexpensive tests to identify the most common drug resistant mutations.

\section{Additional file}

Additional file 1: Table S1. Known HIV drug resistance mutations and resistance in samples from patients with virological failure and slow virological responders at baseline and 6 months.

\section{Abbreviations}

ART: Antiretroviral therapy; HIVDR: HIV drug resistance; NNRTI: Nonnucleoside reverse transcriptase inhibitors; NNRTI: Nucleoside reverse transcriptase inhibitors; SSI: Statens Serum Institut; WHO: World Health Organization; VL: Viral load.

\section{Competing interests}

The authors declare that they have no competing interests.

\section{Authors' contributions}

AAl, HF and ABA conceived the study and designed the study. AAI, DY, MFO, MT, PK and TG took care of participants' recruitment. AAl was responsible for the VL measurements and drafted the manuscript. JF and LHC performed the HIVDR genotype analysis. AAI, ABA, CP, AMA and AAs contributed in the interpretation of results. All authors reviewed and approved the manuscript.

\section{Acknowledgements}

We are grateful to the study participants for their voluntary participation. The Danish International Development Agency (DANIDA) financially supported the study (Grant number: 09-026RH). The authors thank Camilla Dalgaard (Virus Surveillance and Research Section, SSI) for technological assistance with laboratory analyses, Claus Nielsen and Thea Kølsen Fischer (Virus Surveillance and Research Section, SSI) for planning and supervision of the laboratory work. 


\section{Author details}

'Department of Medical Laboratory Sciences \& Pathology, Jimma University, Jimma, Ethiopia. ²Department of Infectious Diseases, Odense University Hospital, Odense, Denmark. ${ }^{3}$ Department of Internal Medicine, Jimma University, Jimma, Ethiopia. ${ }^{4}$ Department of Microbiological Diagnostics \& Virology, Statens Serum Institut, Copenhagen, Denmark. ${ }^{5}$ Department of Nutrition, Exercise and Sports, University of Copenhagen, Copenhagen, Denmark. ${ }^{6}$ Department of Psychiatry, Jimma University, Jimma, Ethiopia. ${ }^{7}$ Department of Pediatrics and Child Health Jimma University, Jimma, Ethiopia. ${ }^{8}$ Armauer Hansen Research Institute, Addis Ababa, Ethiopia.

Received: 3 January 2014 Accepted: 31 March 2014

Published: 4 April 2014

\section{References}

1. CSA: Ethiopian Demographic and Health Survey. Addis Ababa, Ethiopia: Central Statistics Authority of Ethiopia (CSA); 2011.

2. Assefa Y, Jerene D, Lulseged S, Ooms G, Van Damme W: Rapid Scale-Up of Antiretroviral Treatment in Ethiopia: Successes and System-Wide Effects. PLoS Med 2009, 6:e1000056.

3. FMOH: Country Progress Report on HIV/AIDS Response. Addis Ababa, Ethiopia: Federal Ministry of Health of Ethiopia/National HIV/AIDS Prevention and Control Office; 2012

4. Cozzi-Lepri A, Paredes Phillips AN, Clotet B, Kjaer J, Von Wyl V, Kronborg G Castagna A, Bogner JR, Lundgren JD, EuroSIDA in EuroCoord: The rate of accumulation of nonnucleoside reverse transcriptase inhibitor (NNRTI) resistance in patients kept on a virologically failing regimen containing an NNRTI. HIV Med 2012, 13:62-72.

5. Zeh C, Weidle PJ, Nafisa L, Lwamba HM, Okonji J, Anyango E, Bondo P, Masaba R, Fowler MG, Nkengasong JN, Thigpen MC, Thomas T: HIV-1 drug resistance emergence among breastfeeding infants born to HIV-infected mothers during a single-arm trial of triple-antiretroviral prophylaxis for prevention of mother-to-child transmission: a secondary analysis. PLOS Med 2011, 8:e1000430

6. Pillay D: The emergence and epidemiology of resistance in the nucleosideexperienced HIV-infected population. Antivir Ther 2001, 6(Suppl 3):15-24.

7. Ghosn J, Chaix M-L, Delaugerre C: HIV-1 resistance to first- and secondgeneration non-nucleoside reverse transcriptase inhibitors. AIDS ReV 2009, 11:165-173.

8. Bennett DE, Bertagnolio S, Sutherland D, Gilks CF: The World Health Organization's global strategy for prevention and assessment of HIV drug resistance. Antivir Ther 2008, 13(Suppl 2):1-13.

9. Jordan MR, Bennett DE, Wainberg MA, Havlir D, Hammer S, Yang C, Morris L, Peeters M, Wensing AM, Parkin N, Nachega JB, Phillips A, De Luca A, Geng E, Calmy A, Raizes E, Sandstrom P, Archibald CP, Perriëns J, McClure CM, Hong SY, McMahon JH, Dedes N, Sutherland D, Bertagnolio S: Update on World Health Organization HIV drug resistance prevention and assessment strategy: 2004-2011. Clin Infect Dis Off Publ Infect Dis Soc Am 2012, 54(Suppl 4):S245-S249.

10. Descamps D, Chaix M-L, Montes B, Pakianather S, Charpentier C, Storto A, Barin F, Dos Santos G, Krivine A, Delaugerre C, Izopet J, Marcelin A-G, Maillard A, Morand-Joubert L, Pallier C, Plantier J-C, Tamalet C, Cottalorda J, Desbois D, Calvez V, Brun-Vezinet F, Masquelier B, Costagliola D, ANRS AC11 Resistance Study Group: Increasing prevalence of transmitted drug resistance mutations and non-B subtype circulation in antiretroviral-naive chronically HIV-infected patients from 2001 to 2006/2007 in France. J Antimicrob Chemother 2010, 65:2620-2627.

11. Readhead AC, Gordon DE, Wang Z, Anderson BJ, Brousseau KS, Kouznetsova MA, Forgione LA, Smith LC, Torian LV: Transmitted antiretroviral drug resistance in New York State, 2006-2008: results from a new surveillance system. Plos One 2012, 7:e40533.

12. Audelin AM, Lohse N, Obel N, Gerstoft J, Jørgensen LB: The incidence rate of HIV type-1 drug resistance in patients on antiretroviral therapy: a nationwide population-based Danish cohort study 1999-2005. Antivir Ther 2009, 14:995-1000

13. FMoH: National Guidelines for HIV/AIDS and Nutrition in Ethiopia. Addis Ababa, Ethiopia: Federal Ministry of Health of Ethiopia (FMoH); 2008.

14. WHO: Antiretroviral therapy for HIV infection in adults and adolescents: Recommendations for a public health approach. Geneva: World Health Organization; 2006. Available from: http://www.who.int/hiv/pub/guidelines/ artadultguidelines.pdf.
15. Lindström A, Albert J: A simple and sensitive "in-house" method for determining genotypic drug resistance in HIV-1. J Virol Methods 2003, 107:45-51.

16. Vandamme AM, Witvrouw M, Pannecouque C, Balzarini J, Van Laethem K, Schmit JC, Desmyter J, De Clercq E: Evaluating Clinical Isolates for Their Phenotypic and Genotypic Resistance Against Anti-HIV Drugs. Methods Mol Med 2000, 24:223-258.

17. Madsen TV, Leitner T, Lohse N, Obel N, Ladefoged K, Gerstoft J, Petersen AB, Nielsen C, Jørgensen LB: Introduction of HIV type 1 into an isolated population: molecular epidemiologic study from Greenland. AIDS Res Hum Retroviruses 2007, 23:675-681.

18. Liu TF, Shafer RW: Web resources for HIV type 1 genotypic-resistance test interpretation. Clin Infect Dis Off Publ Infect Dis Soc Am 2006, 42:1608-1618.

19. Barth RE, van der Loeff MFS, Schuurman R, Hoepelman AIM, Wensing AMJ: Virological follow-up of adult patients in antiretroviral treatment programmes in sub-Saharan Africa: a systematic review. Lancet Infect Dis 2010, 10:155-166.

20. Kassu A, Fujino M, Matsuda M, Nishizawa M, Ota F, Sugiura W: Molecular epidemiology of HIV type 1 in treatment-naive patients in north Ethiopia. AIDS Res Hum Retroviruses 2007, 23:564-568.

21. Abegaz WE, Grossman Z, Wolday D, Ram D, Kaplan J, Sibide K, Wuhib T, Ismael S, Nkengasong J, Mekonen T, Berhanu H, Messele T, Lulseged S, Maayan S, Mengistu Y: Threshold survey evaluating transmitted HIV drug resistance among public antenatal clinic clients in Addis Ababa, Ethiopia. Antivir Ther 2008, 13(Suppl 2):89-94.

22. WHO: WHO HIV Drug Resistance Report 2012 HIV/AIDS Programme. Geneva: WHO; 2012

23. Biressaw S, Abegaz WE, Abebe M, Taye WA, Belay M: Adherence to Antiretroviral Therapy and associated factors among HIV infected children in Ethiopia: unannounced home-based pill count versus caregivers' report. BMC Pediatr 2013, 13:132

24. Rusine J, Asiimwe-Kateera B, van de Wijgert J, Boer KR, Mukantwali E, Karita E, Gasengayire A, Jurriaans S, de Jong M, Ondoa P: Low primary and secondary HIV drug-resistance after 12 months of antiretroviral therapy in human immune-deficiency virus type 1 (HIV-1)-infected individuals from Kigali. Rwanda. PloS One 2013, 8:e64345.

25. Liégeois F, Vella C, Eymard-Duvernay S, Sica J, Makosso L, Mouinga-Ondémé A, Mongo AD, Boué V, Butel C, Peeters M, Gonzalez J-P, Delaporte E, Rouet F: Virological failure rates and HIV-1 drug resistance patterns in patients on first-line antiretroviral treatment in semirural and rural Gabon. J Int AIDS SOC 2012, 15:17985

26. Hamers RL, Schuurman $R$, Sigaloff $K C E$, Wallis $C L$, Kityo $C$, Siwale M, Mandaliya K, Ive P, Botes ME, Wellington M, Osibogun A, Wit FW, van Vugt M, Stevens WS, de Wit TFR, PharmAccess African Studies to Evaluate Resistance (PASER) Investigators: Effect of pretreatment HIV-1 drug resistance on immunological, virological, and drug-resistance outcomes of first-line antiretroviral treatment in sub-Saharan Africa: a multicentre cohort study. Lancet Infect Dis 2012, 12:307-317.

27. Wittkop L, Günthard HF, de Wolf F, Dunn D, Cozzi-Lepri A, de Luca A, Kücherer C, Obel N, von Wyl V, Masquelier B, Stephan C, Torti C, Antinori A García F, Judd A, Porter K, Thiébaut R, Castro H, van Sighem Al, Colin C, Kjaer J, Lundgren JD, Paredes R, Pozniak A, Clotet B, Phillips A, Pillay D, Chêne G, EuroCoord-CHAIN study group: Effect of transmitted drug resistance on virological and immunological response to initial combination antiretroviral therapy for HIV (EuroCoord-CHAIN joint project): a European multicohort study. Lancet Infect Dis 2011, 11:363-371.

28. Weber J, Chakraborty B, Weberova J, Miller MD, Quiñones-Mateu ME: Diminished Replicative Fitness of Primary Human Immunodeficiency Virus Type 1 Isolates Harboring the K65R Mutation. J Clin Microbiol 2005, 43:1395-1400

29. Cong M, Heneine W, García-Lerma JG: The Fitness Cost of Mutations Associated with Human Immunodeficiency Virus Type 1 Drug Resistance Is Modulated by Mutational Interactions. J Virol 2007, 81:3037-3041.

doi:10.1186/1471-2334-14-181

Cite this article as: Abdissa et al.: Drug resistance in HIV patients with virological failure or slow virological response to antiretroviral therapy in Ethiopia. BMC Infectious Diseases 2014 14:181 